\title{
Electron Tomography to Analyze Vesiculation in Plant Endosomes
}

\author{
Kaija Goodman, Marisa Otegui and Janice Pennington \\ University of Wisconsin - Madison, Wisconsin, United States
}

The flow of proteins at the plasma membrane is dynamically regulated and crucial for a cell to fine-tune its downstream signaling pathways. Degradative sorting of ubiquitinated plasma membrane proteins occurs at endosomes, where a cargo's fate is determined. First ubiquitinated cargo, such as signaling receptors, transporters, lipids, and other important molecules, are recognized and internalized by endocytosis. Endocytic vesicles traffic to the early endosome where cargo can be deubiquitinated and recycled back to the cytoplasm or continue to be sorted into late endosomes, called multivesicular endosomes. Multivesicular endosomes arise from the invagination of the endosomal limiting membrane to form internal vesicles, sequestering cargo within. Upon fusion with the lysosome/vacuole, cargo proteins are delivered to the lumen for degradation.

The highly-conserved protein machinery, ESCRT (Endosomal Sorting Complex Required for Transport), recognizes and concentrates cargo proteins to form the internal vesicles of multivesicular endosomes. It is the only characterized protein machinery that mediates negative membrane deformation (i.e. membrane bending away from the cytoplasm). During vesiculation, ESCRT loses contact with their cargo, unlike other coat-mediated vesiculation events, such as clathrin-mediated endocytosis, in which coat and adapter proteins remain in contact with their cargo during the whole process. It is also presumed that ESCRT is removed from the membrame and recycled back to the cytoplasm prior to vesicle release (Babst et al., 2002). Long-standing questions remain on the mechanism of membrane fission and how multivesicular endosomes sequester cargo without its escape into the connecting endosomal limiting membrane (Fabrikant et al., 2009; Henne et al., 2012; Chiaruttini et al., 2015; Schöneberg et al., 2017). Moreover, it is a challenge to visualize this highly dynamic process in small mobile endosomal compartments (250 $\mathrm{nm})$, at the edge of light detection, with even smaller internal vesicles (40 nm).

Plants possess similarly conserved function of multivesicular endosomes and have also evolved plantspecific ESCRT function. We are using TEM and electron tomographic analysis of multivesicular endosomes, to understand the structure-function relationship of ESCRT-mediated vesiculation within specific plant tissues and across plant lineage. Coupling electron tomography with computational modeling, we found that multivesicular endosomes in plants have adapted a novel mechanism to entrap cargo more efficiently by forming concatenated networks of vesicles (Buono et al., 2017), which is in contrast to the well-established dogma that ESCRT forms vesicles one by one. In addition, ESCRT components are internalized and kept at inter-vesicular bridges, creating a diffusion barrier for cargo within the vesicle networks. Mutations in several ESCRT subunits compromises vesicle concatenation, cargo retention, and results in aberrant endosome morphology and the misregulation of the abundance and flow of key plasma membrane proteins important for growth and development (Spitzer et al., 2009; Buono et al., 2016).

While tomography only provides a static snapshot of multivesicular endosomes, we are using the vesicle network architecture to model how these dynamic networks form and determine the rules that control vesicle concatenation. We have observed an array of differently sized networks associated with one or multiple connected budding sites on the surface of the endosomal limiting membrane. Examining complex networks, such as two vesicles connected by a lateral bridge at neighboring bud sites, we can apply constraints to create the geometries visualized in the vesicle networks while considering budding activity 
and the loss and/or gain of bud sites to explain network growth. There is a wealth of information within tomographic reconstructions that is accessible with the integration of experimental and computational data, which we are using to define how vesicle networks within plant endosomes are formed.

\section{References}

Babst, M. et al. (2002). Dev. Cell 3: 271-282.

Buono, R.A. et al. (2016). Plant Physiol. 171: 251-64.

Buono, R.A. et al. (2017). JCB 216: 2167-2177.

Chiaruttini, N. et al. (2015). Cell 163: 866-879.

Fabrikant, G. et al. (2009). PLoS Comput. Biol. 5: e1000575.

Henne, W.M. et al. (2012). Cell 151: 356-371.

Spitzer, W.C. et al. (2009). Plant Cell 21: 749-766.

Schöneberg, J. et al. (2017). Nat. Rev. Mol. Cell Biol. 18: 5-17. 